\title{
Large Scale Corpus Analysis and Recent Applications
}

\author{
Yuji Matsumoto \\ Nara Institute of Science and Technology, Japan
}

\begin{abstract}
Recent progress of corpus and machine learning-based natural language processing methodologies have made it possible to handle large scale corpus with a quite high accuracy. The speaker is now involved in a project for constructing a large scale contemporary Japanese balanced corpus, aiming at constructing automatic annotation tools on various levels of natural language analyses. I will first introduce our activities on corpus based natural language analyzers for word dependency parsing and anaphora resolution and annotated corpus management environment. Then, I will explain recent natural language applications such as sentiment/opinion mining and knowledge extraction from a large scale text data like Weblogs.
\end{abstract}

